Math. Model. Nat. Phenom.

Vol. 6, No. 6, 2011, pp. 245-259

DOI: $10.1051 / \mathrm{mmnp} / 20116613$

\title{
Compartmental Models of Migratory Dynamics
}

\author{
J. Knisley ${ }^{1,2}$ *, T. Schmickl ${ }^{3}$, and I. Karsai ${ }^{1,4}$ \\ ${ }^{1}$ The Institute for Quantitative Biology, East Tennessee State University \\ ${ }^{2}$ Mathematics and Statistics, East Tennessee State University, Johnson City, TN 37614 USA \\ ${ }^{3}$ Artificial Life Lab of the Department of Zoology, Karl-Franzens-University \\ ${ }^{4}$ Biological Sciences, East Tennessee State University, Johnson City, TN 37614 USA
}

\begin{abstract}
Compartmentalization is a general principle in biological systems which is observable on all size scales, ranging from organelles inside of cells, cells in histology, and up to the level of groups, herds, swarms, meta-populations, and populations. Compartmental models are often used to model such phenomena, but such models can be both highly nonlinear and difficult to work with.

Fortunately, there are many significant biological systems that are amenable to linear compartmental models which are often more mathematically accessible. Moreover, the biology and mathematics is often so intertwined in such models that one can be used to better understand the other. Indeed, as we demonstrate in this paper, linear compartmental models of migratory dynamics can be used as an exciting and interactive means of introducing sophisticated mathematics, and conversely, the associated mathematics can be used to demonstrate important biological properties not only of seasonal migrations but also of compartmental models in general.

We have found this approach to be of great value in introducing derivatives, integrals, and the fundamental theorem of calculus. Additionally, these models are appropriate as applications in a differential equations course, and they can also be used to illustrate important ideas in probability and statistics, such as the Poisson distribution.
\end{abstract}

Key words: compartmental models, erlang, poisson, exponential AMS subject classification: 34A30, 62P10, 92B05, 97M10

\footnotetext{
${ }^{*}$ Corresponding author. E-mail: knisleyj@etsu.edu
} 


\section{Introduction}

Mathematical models and dynamical systems are becoming increasingly important in biology education [5, 14], as are realistic representations of biological phenomena [6]. In ecology, for instance, such realistic representations have a long history of being implemented with compartmental modeling. Similarly, compartmental modeling is both important and increasing in importance in biology, medicine, pharmacology, and a number of the other life sciences [28], often as a pathway into systems biology [1]. However, the mathematics associated with these applications is not always student friendly, especially when the models meet the need for meaningful quantitative applications [9, 27].

Fortunately, there are linear compartmental models which are not only important in the biosciences, but are also mathematically accessible, even for undergraduate students with limited backgrounds. For example, linear compartmental models are effective in modeling the kinetics of a tracer through a system that is in steady state [8] and have been used in conjunction with tracer experiments at the whole-body, organ, and cellular level [17]. Linear compartmental models are also used in epidemiology, in proteomics, in ecology, and elsewhere [10, 22, 25].

Conversely, the biological contexts that utilize linear compartmental models can be used to introduce and develop many aspects of differential and integral calculus [20]. Indeed, many linear compartmental systems are analogous to the biological context of migratory models [3], and there are migratory models that are intuitive, accessible, and amenable to highly visual, "hands-on" exploration.

In this paper, we focus on the annual juvenile salmon migrations toward the oceans. Each year, adult salmon swim from the ocean upstream to mouths of the rivers where they were spawned, and once there each surviving female lays thousands of eggs. The eggs hatch into aleven, grow into fry, and subsequently enter a process of "smoltification" in which they transform physiologically from fresh water to saltwater fish. They then swim downstream to the nutrient-rich open ocean where they develop into adults [12].

However, these annual migrations have been compromised, often severely, by the construction of dams and other man-made features, thus greatly adding to the naturally large mortality rates of the smolt populations and creating the need for mathematical models which incorporate mortality into the migrations [4, 29]. In their simplest forms, such models can be highly appropriate for students with little or no mathematical preparation, thus providing an intuitive context in which to introduce important mathematical ideas. Subsequently, as the models increase in size and scope - and as the level of student preparation increases - the associated mathematical concepts acquire rigorously accurate, highly intuitive interpretations that are beneficial to the biology.

We begin by discussing a simple, single-compartmental model, which correspondingly allows us to intuitively introduce important ideas in calculus, as well as in probability. We then progress to a full multi-compartmental model with progressively more sophisticated mathematics [11]. Ultimately, we transition from the value of the biological context in introducing mathematical concepts to the value in using mathematics to better understand the associated biology. Throughout, we discuss how this material can be (and has been) used to introduce and explore concepts in calculus 
and probability that are important throughout quantitative biology and biology education.

\section{Compartmental Modeling of Idealized Migrations}

\subsection{Exploring the Biological Context}

Typically, we introduce these models by dividing a river into a contiguous collection of habitat zones and assuming that the population is initially in zone 0 , which corresponds to the river segment where the salmon hatched and developed into smolt. After living in zone 0 for almost a year, the smolt will actively swim down the river into the ocean estuaries, which corresponds in our model to moving independently at the same rate, on average, through the sequence of zones (i.e., compartments).

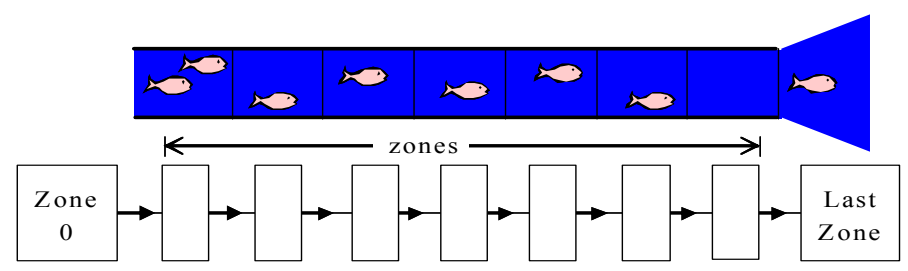

Figure 1: Habitat zones between hatching site (zone 0) and the ocean (last zone)

Thus, if $N(t)$ is the population of the habitat zone at time $t$ in days, then the population one day later satisfies

$$
N(t+1)=N(t)+\text { Arrivals - Departures },
$$

where the arrivals and departures are those entering or leaving, respectively, during that day.

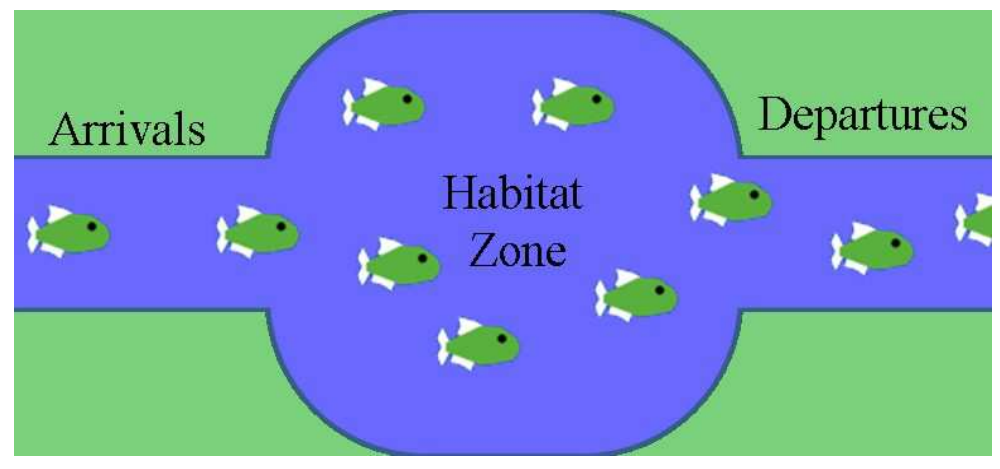

Figure 2: Habitat zone with population $N(t)$ at time $t$.

More generally, arrivals and departures may be in units of individuals per hour or individuals per minute rather than individuals per day. Consequently, if $\Delta t$ is the time increment in question - 
i.e., the next day, the next hour, etcetera - then arrivals are at an immigration rate of I individuals per unit time, or equivalently,

Arrivals during a given time increment $=I \cdot \Delta t$.

Likewise, Departures $=E \cdot \Delta t$, where $E$ is the rate of emigration, so that the model of the habitat zone population is, in general,

$$
N(t+\Delta t)=N(t)+I \Delta t-E \Delta t .
$$

\subsection{Motivating Concepts in Calculus}

Conversely, this model allows us to introduce concepts in calculus in an intuitive fashion. For example, there is no emigration out of the final habitat zone - i.e., the Ocean - other than a relatively small number, $\varepsilon>0$, of "removals" per unit time. Thus, the model for the ocean is given by

$$
N(t+\Delta t)=N(t)+I \Delta t-\varepsilon \Delta t .
$$

A natural question is that of how to measure the rate $I$ at which the smolts are entering the ocean, especially if $I=I(t)$ is a function of $t$ itself (changing over time). Solving for $I$ in (2.1) leads to

$$
I(t)=\frac{N(t+\Delta t)-N(t)}{\Delta t}+\varepsilon
$$

Supposing now that the removal rate $\varepsilon$ is so small that it approaches 0 as $\Delta t$ itself approaches 0 , we have

$$
I(t)=\lim _{\Delta t \rightarrow 0} \frac{N(t+\Delta t)-N(t)}{\Delta t},
$$

where the notation $\lim _{\Delta t \rightarrow 0}$ means the result obtained by letting $\Delta t$ become an arbitrarily small time increment (minutes, seconds, milliseconds, etcetera). We now have the rate of change, $I(t)$, of the population given by the definition of the derivative.

Similarly, if $T=n \Delta t$ for some very large $n$ - typically, $T$ is about 1 month [12] whereas $\Delta t$ is in days or minutes - then the question of the total number of smolts entering the ocean (i.e., ignoring removals, for now ) leads us to notice that

$$
\begin{aligned}
& N\left(t_{0}+\Delta t\right)=N\left(t_{0}\right)+I\left(t_{0}\right) \Delta t=N(0)+I\left(t_{0}\right) \Delta t, \\
& N\left(t_{1}+\Delta t\right)=N\left(t_{1}\right)+I\left(t_{1}\right) \Delta t=N(0)+I\left(t_{0}\right) \Delta t+I\left(t_{1}\right) \Delta t,
\end{aligned}
$$

and so on, where $t_{k}=t_{0}+k \cdot \Delta t$. Continuing in the same fashion, we find that the number of smolts entering the ocean between time 0 and time $T$ is

$$
N(T)=N(0)+\sum_{k=0}^{n-1} I\left(t_{k}\right) \Delta t
$$


Again, allowing $\Delta t$ to approach 0 leads to

$$
N(T)-N(0)=\int_{0}^{T} I(t) d t=\int_{0}^{T} N^{\prime}(t) d t,
$$

which is the fundamental theorem of calculus.

Additionally, it is easy to justify - indeed, the core of engineers uses this concept - that we need not use the same $\Delta t$ for every increment. Early and late in the migration, units of individuals per day are more than sufficient, whereas at times in between it may be necessary to change to units of individuals per hour or minute. In this way, we can argue that a correct definition of the integral should be

$$
\int_{0}^{T} I(t) d t=\lim _{\Delta t \rightarrow 0} \sum_{k=0}^{n-1} I\left(t_{k}\right) \Delta t_{k},
$$

where $\Delta t_{k} \leq \Delta t$ for all $k=1, \ldots, n$. Given sufficient technology via simulations and data, these notions of derivative and integral can be made highly intuitive within this context.

\section{A One Compartment Model for Zone 0}

\subsection{Exponential Decay and the Exponential Distribution}

When the smolt migration begins, the majority of the juvenile salmon are in the streams where they were spawned. We refer to these habitats collectively as zone 0 , and given students with a sufficient calculus background, then the discussion can move to a level of sophistication appropriate for a first modeling course, such as those represented by textbooks like [2] or [25].

Based on physiological and environmental factors the smolt population starts a very synchronized migration downriver, often leaving zone 0 just a few days after the migration starts.

We assume that each individual randomly emigrates from zone 0 , so that if $N_{0}(t)$ is the size of the population at time $t$ (typically, in days) and if $m$ is the constant per capita rate of migration, then the population at some small increment $\Delta t$ since time $t$ satisfies

$$
N_{0}(t+\Delta t) \approx N_{0}(t)-(m \Delta t) \cdot N_{0}(t)
$$

where the approximations $(\approx)$ become exact as $\Delta t$ approaches zero. ${ }^{\dagger}$ Typically, we simulate this behavior by imagining zone 0 to be a "lake" with fish swimming quickly enough that they randomly encounter the mouth of the adjoining river and begin their journey to the ocean.

We also show that if the initial population of zone 0 is denoted by $K$, then

$$
N_{0}(\Delta t) \approx K(1-m \Delta t), \quad N_{0}(2 \Delta t) \approx K(1-m \Delta t)^{2},
$$

\footnotetext{
${ }^{\dagger}$ When working with students, we tend to use either $\Delta_{t}$ - or even just $\Delta-$ to denote the time increment $\Delta t$.
} 


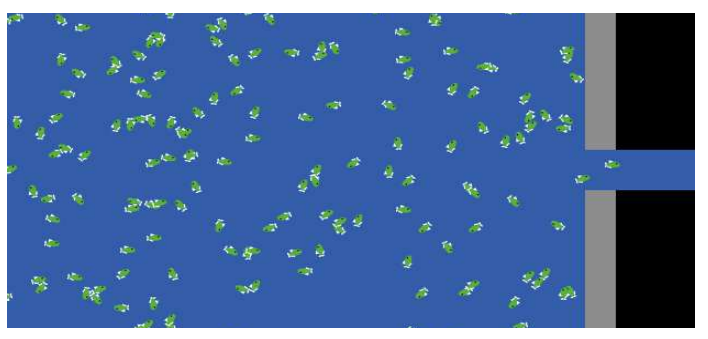

Figure 3: An agent based simulation of zone 0 (Netlogo)

and etcetera, so that for any positive integer $j$ we have $N_{0}(j \Delta t) \approx K(1-m \Delta t)^{j}$. Since $t=j \Delta t$ is the result of $j$ time steps, we have

$$
N_{0}(t) \approx K\left(1-\frac{m t}{j}\right)^{j},
$$

which in the limit as $\Delta t \rightarrow 0$ (i.e. $j \rightarrow \infty$ ) leads to

$$
N_{0}(t)=K e^{-m t} .
$$

After exploring this model via simulations and other activities, it may be appropriate to introduce an alternative interpretation - i.e., a probabilistic interpretation - of the model. For each $t>0$, the proportion of the population remaining in the lake at time $t$ is

$$
p r(t)=\frac{K e^{-m t}}{K}=e^{-m t} .
$$

Consequently, if we interpret the probability that a fish chosen at random remains in the lake until time $t$ to be equal to the proportion of the overall population that remains at time $t$, then

$$
\operatorname{Pr}(\text { waiting until time } t)=e^{-m t},
$$

and conversely, the probability that a fish leaves the lake by time $t$ is

$$
\operatorname{Pr}(\text { departure by time } t)=1-e^{-m t} \text {. }
$$

Alternatively, if $t$ is fixed, then $\mu=m t$ can be interpreted as the average number of departures from zone 0 between times 0 and $t$, which means that $e^{-\mu}$ is the probability that a fish chosen at random remains in zone 0 after $t$ days. 
Simulations in Netlogo can be used to illustrate and explore this probabilistic interpretation [30]. In these simulations, the physiological and environmental factors influencing the rate of migration are modeled by a "relative flow rate" [19]. For example, if initially there are 100 individuals with a per capita emigration rate of $100 \%$ per day, then there will be approximately $100 e^{-1} \approx 37$ fish remaining after one day, approximately $100 e^{-2} \approx 13.534$ after 2 days, and so on. Or equivalently, the simulation for a single marked fish can be run repeatedly from time 0 to time $t$, and then the proportion of the total trials in which the marked fish remains can be calculated.

In addition, the simple form of this model allows us to explore real-world data, such as data from the Fish Passage Center (www.fpc.org) which measures fish populations over time at various locations along the Columbia river and its tributaries. Finally, the derivation of (3.2) allows us to motivate the biologically important Bernoulli Approximations, which say that if $a$ is sufficiently close to 0 and if $N$ is sufficiently large, then

$$
(1+a)^{N} \approx e^{N a}, \quad(1-a)^{N} \approx e^{-N a} .
$$

These approximations are used frequently in biology - for example, in population genetics [15].

\section{A Multi-compartment Migratory Model}

\subsection{The First Two Zones}

Students who have sufficiently acquired the material for zone 0 will soon realize that fish leaving zone 0 are migrating into zone 1 - and they are entering zone 1 at the same per capita rate $m$ at which they left zone 0 . Consequently, if $N_{1}(t)$ is the population of zone 1 at time $t$ and if fish are

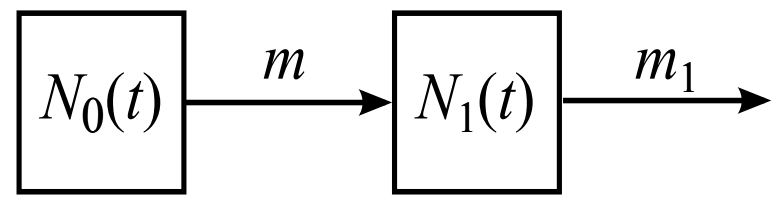

Figure 4: The migration model for zone 1

leaving zone 1 (and entering zone 2) at a per capita rate of $m_{1}$, then

$$
\begin{aligned}
\text { Next } & \approx \text { Now - Emigration + Immigration } \\
N_{1}(t+\Delta t) & \approx N_{1}(t)-\left(m_{1} \Delta t\right) N_{1}(t)+(m \Delta t) N_{0}(t) .
\end{aligned}
$$

That is, a percentage $m \Delta t$ of the population $N_{0}(t)$ in zone 0 enter into zone 1 over a period of $\Delta t$ days, while a proportion $m_{1} \Delta t$ of $N_{1}(t)$ depart. After some algebraic manipulation and a limit, we have the following differential equation model for zone 1 :

$$
\frac{d N_{1}}{d t}=-m_{1} N_{1}+m N_{0}
$$


If $m_{1} \neq m$, then the initial condition $N_{1}(0)=0$ implies a solution of the form

$$
N_{1}(t)=\frac{m K}{m-m_{1}}\left(e^{-m_{1} t}-e^{-m t}\right) .
$$

However, we tend to de-emphasize this case because it becomes exponentially more complicated as the number of compartments increases and because if $m_{1}$ is even crudely approximately the same as $m$, then the case $m_{1}=m$ produces a solution that is qualitatively very similar (see Figure 5 ).

Instead, we motivate $m_{1}=m$ by similar flow rates, similar fish speeds, etcetera. Subsequently, we use the form $N_{0}=K e^{-m t}$ to motivate a "guess" of $N_{1}(t)=C t e^{-m t}$ for some unknown constant $C$, and then we substitute into the differential equation (4.1) to determine $C$. After simplifying, we find that

$$
N_{1}(t)=m t K e^{-m t} .
$$

Although not similar algebraically to the solution (4.2) in which $m_{1} \neq m$, Figure 5 illustrates that the solutions are qualitatively very similar even if $m_{1}$ is only very roughly the same as $m$.

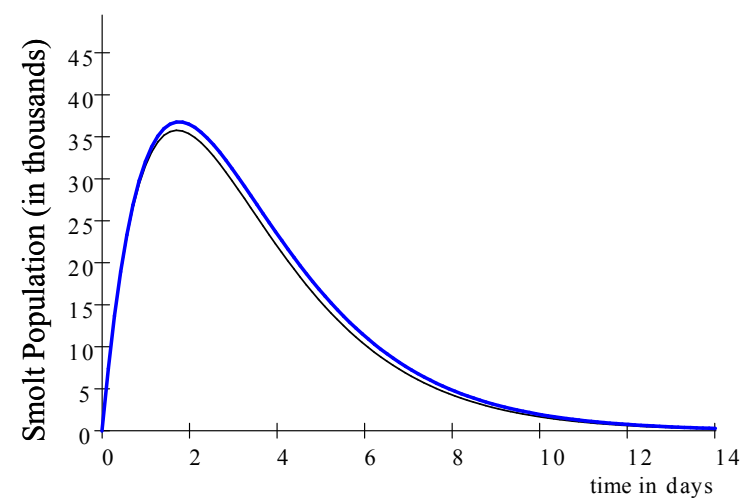

Figure 5: Zone 1 population for $m_{1}=0.6, m=0.568$ (black) and for $m_{1}=m=0.568$ (blue).

\subsection{Multiple Zones and the Poisson Distribution}

More generally, given a sequence of zones (compartments), we can use the same process used for zone 1 to explore the solutions for subsequent zones.

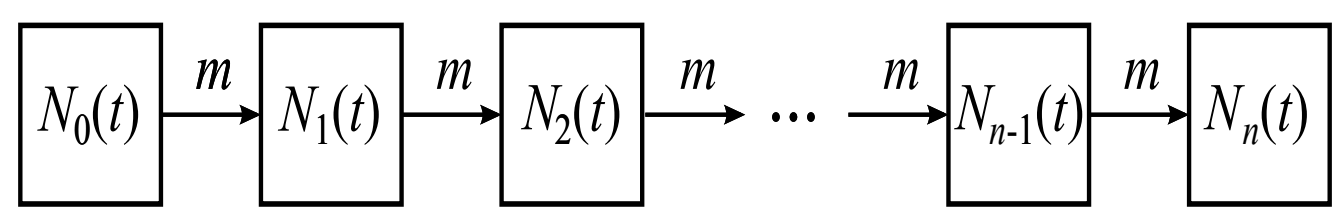

Figure 6: The entire compartmental model of migration down a river to the sea. 
To begin with, if $N_{j}(t)$ is the population of zone $j$ at time $t$, then the discussion used for zone 1 applied to zone $j$ leads to

$$
\frac{d N_{j}}{d t}=-m_{j} N_{j}+m_{j-1} N_{j-1} .
$$

Again, we motivate an assumption of $m=m_{1}=m_{2}=\ldots=m_{j}$, so that (4.3) is an Erlang differential equation (whose solution leads to the Erlang distribution).

Inductively, we use $N_{1}(t)=(m t) K e^{-m t}$ to motivate us to guess that $N_{2}(t)=C(m t)^{2} K e^{-m t}$ for some unknown constant $C$. Substituting and solving for $C$ leads to

$$
N_{2}(t)=K \frac{(m t)^{2}}{2} e^{-m t} .
$$

Similarly, using the form of $N_{2}(t)$ along with the product rule leads us to show that

$$
N_{3}(t)=K \frac{(m t)^{3}}{3 !} e^{-m t}
$$

and so on, so that for each $j=0, \ldots, n-1$., we have

$$
N_{j}(t)=K \frac{(m t)^{j}}{j !} e^{-m t} .
$$

This sequence of solutions is illustrated in Figure 7.

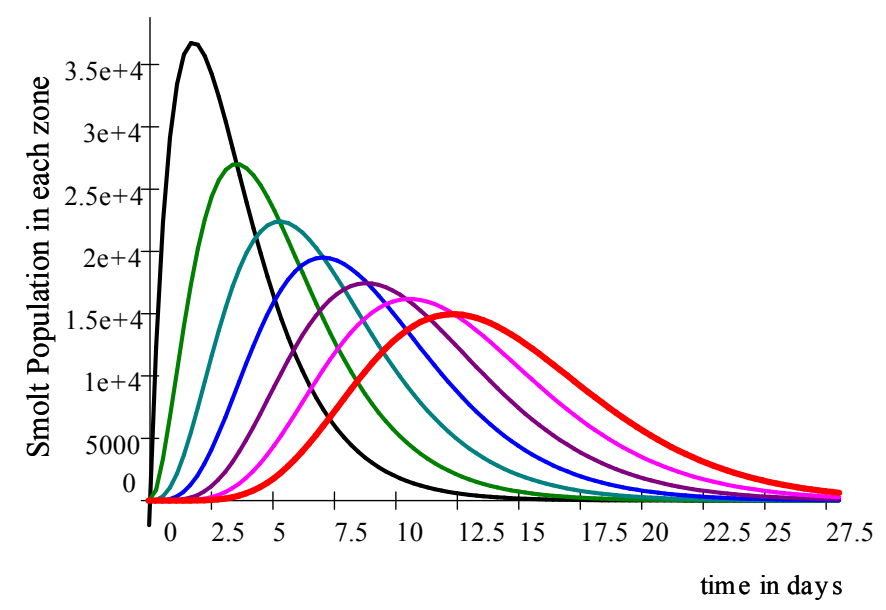

Figure 7: Solutions for zone 1 (black), zone 2 (green), zone 3 (aqua), zone 4 (blue), zone 5 (purple), zone 6 (magenta), and zone 7 (red).

As we develop the mathematics for the idealized migration model, we also explore it via interactive simulations. In particular, the students can watch individual fish as they swim through the sequence of zones (compartments). Consequently, after sufficient exposure to the mathematics 
and to the simulations, we note that for fixed $t$, the quantity $\mu=m t$ is the average number of fish remaining in zone 0 at time $t$. Consequently, the solution

$$
N_{j}(t)=K \frac{\mu^{j}}{j !} e^{-\mu}
$$

is the number of fish remaining in zone $j$ at time $t$. The proportion of the original population of size $K$ located in zone $j$ at time $t$ is thus

$$
p r=\frac{\mu^{j}}{j !} e^{-\mu} .
$$

If we define individual "success" to be that of moving from one zone to the next, then being in zone $j$ is equivalent to " $j$ successes." This means that if $X$ is the random variable representing the number of successes of a fish chosen at random, then

$$
\operatorname{Pr}(X=j)=\frac{\mu^{j}}{j !} e^{-\mu},
$$

which is the Poisson distribution. Thus, our idealized migration model leads to a highly intuitive, mathematically defensible introduction to the Poisson distribution.

Correspondingly, this context allows the introduction and exploration of associated concepts, such as the central limit theorem, the normal distribution, and the Poisson approximation of the normal. In particular, as shown by the red curve in Figure 7 and as is apparent in the interactive models, small variations in individual behavior tend to lead to a "bolus" movement of the "herd" (i.e., the population). Consequently, by the time the population reaches zone $j$ for $j$ sufficiently large, it is approximately normally distributed in time. It is a beneficial exercise to have students find the maximum and inflection points of $N_{j}(t)$ and thus obtain the "mean" and "standard deviation" of the corresponding normal distribution.

\section{Incorporating Mortality}

The last habitat zone does not have any emigration and at this point, also has no reproduction or mortality, so that the total population of the system is fixed at $K$. Thus, the population of the last habitat zone is $K$ less the sum of all the smolts in all the zones except the last one:

$$
N_{n}(t)=K-\sum_{j=0}^{n-1} N_{j}(t)=K-K \sum_{j=0}^{n-1} \frac{m^{j} t^{j}}{j !} e^{-m t} .
$$

This allows us to construct a full model of the idealized migration, and it also allows us to discuss mortality in the migration models.

In particular, mortality is incorporated by assuming that each habitat zone has two emigration terms-one to the next habitat zone at rate $m$ and one to a "zone" called the removeds at a per capita 


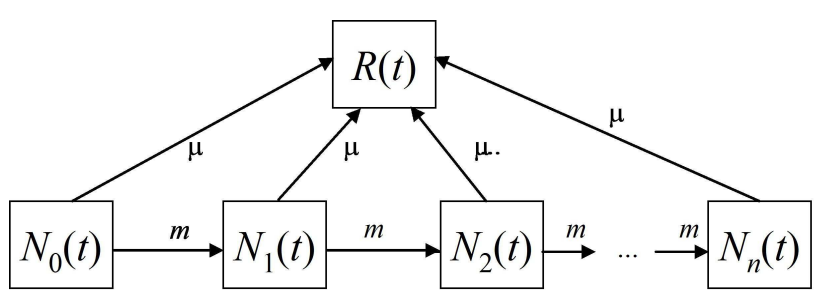

Figure 8: $R(t)$ is the number removed from the population at time $t$.

death rate $\mu$ for each zone. (This is conceptually similar to classic SIR epidemiological models, in which the "R" compartment represents those "removed" from the infected population by either recovery or mortality).

If we assume density dependent mortality, then the the total number $R(t)$ removed from the system by time $t$ satisfies the differential equation $R(t)$ is

$$
\frac{d R}{d t}=\mu N_{0}+\mu N_{1}+\ldots+\mu N_{n}=\mu\left(N_{0}+N_{1}+\ldots+N_{n}\right) .
$$

However, since the population is size $K$ initially, there must be $K$ smolts in the system at all times, so that

$$
N_{0}(t)+N_{1}(t)+\ldots+N_{n}(t)+R(t)=K
$$

and thus, $N_{0}+N_{1}+\ldots+N_{n}=K-R$. It follows that

$$
\frac{d R}{d t}=\mu\left(N_{0}+N_{1}+\ldots+N_{n}\right)=\mu(K-R(t)) .
$$

That is, no matter how many zones are in the system, the removeds satisfy the differential equation

$$
\frac{d R}{d t}=\mu K-\mu R,
$$

which has a solution of

$$
R(t)=K-K e^{-\mu t} .
$$

Similar to above, it can also be shown that the solution for the $j^{\text {th }}$ zone is

$$
N_{j}(t)=K \frac{m^{j} t^{j}}{j !} e^{-(m+\mu) t}, \quad j=0, \ldots, n-1 .
$$




\section{Conclusions}

Although we have focused on a particular migratory model in this paper, the same approach can be used in a number of biological contexts. Specifically, linear compartmental models can be used, as a starting point at least, in the study of other migratory populations, including the huge herring swarms that migrate every year, the gnu-mass migration ins the African savanna, the migratory birds, the whales moving between Hawaii and the Arctic, the "Acherontia atropos" moth migrating throughout Europe, and the giant-honeybee colonies that move across India every year, to name a few [13].

Moreover, in pharmacokinetic studies, many agents - drugs, tracers, etcetera - are assumed to flow at a relatively fixed rate through a linear compartmental system, where the compartments are organs or systems or even cells [17]. Often such systems are studied only qualitatively or via numerical simulations. However, as this paper illustrates, the concept of "habitat zones" can be re-interpreted as pharmacokinetic compartments, thus allowing $N_{j}(t)$ to be interpreted as the tracer concentration at time $t$ in compartment $j$. Correspondingly, the Poisson-distribution like solutions described above are instead closed form solutions of the pharmacokinetic system itself. In the very least, obtaining closed form solutions instead of individual numerical simulations can be used to illustrate the biological value in analytical approaches to mathematically sophisticated models.

Moreover, this modeling approach also has value mathematically, if only in motivating the same mathematics in contexts ranging from Erlang distributions in protein-protein interactions [16] to the development of Poisson models in the study of fossils [26]. There are also many other aspects of this model which can be explored. For example, we did not discuss the fact that $K$ is an equilibrium of the last habitat zone, the motivation for statistical testing of the goodness of fit of the model to migratory data, or the use of multi-variable Taylor's series to explore how sensitive the model is to variations in the parameters $m_{j}$.

Admittedly, these models are highly idealized. However, appropriate biological contexts for introducing mathematical ideas are of significant pedagogical importance [21]. Mathematical models provide templates where data, mechanisms, simulations, and theories are represented in a uniform and transparent fashion which makes it easy for members of interdisciplinary teams to collaborate [18]. Correspondingly, the development of mathematical pedagogy within suitable biological contexts has been one of the primary goals of the Symbiosis project [24]. Indeed, as first models - and especially as pedagogical tools - we have found the modeling of migration to not only be valuable biologically, but also of significant value mathematically, even serving as a canonical illustration of biological systems in much the same way that the harmonic oscillator serves as a canonical example in physics.

\section{Acknowledgments}

The authors wish to thank all the members of the Symbiosis project for the many fruitful discussions and assistance in developing this model. This work is supported by a major grant from the Howard Hughes Medical Institute (Grant \# 52005872). The authors would also like to thank the 
reviewers and editors for their excellent suggestions for improving upon the initial submission of this manuscript.

\section{References}

[1] M. Allarakhia, A. Wensley. Systems biology: melting the boundaries in drug discovery research. Technology Management: A Unifying Discipline for Melting the Boundaries, (2005), 262-274.

[2] L. Allen. An Introduction to mathematical biology, Pearson, New York, 2007.

[3] S. Bauer, Z. Barta, B. Ens, G. Hays, J. McNamara, M. Klassen. Animal migration: linking models and data beyond taxonomic limits. Biol. Lett., 5 (2009), No. 4, 433-435.

[4] R. Baxter. Environmental effects of dams and impoundments. Ann. Rev. Ecol. Syst., 8 (1977), 255-93.

[5] W. Bialek, D. Botstein. Introductory science and mathematics education for the 21th century biologists. Science, 303 (2004), 788-790.

[6] J. Bower. Looking for Newton: realistic modeling in modern biology. Brains, Minds and Media, 1 (2005), bmm217 (urn:nbn:de:0009-3-2177).

[7] V. Buonaccorsi, A. Skibiel, A striking demonstration of the Poisson distribution. Teach. Stat, 27 (2005), 8-10.

[8] C. Cobelli. Modeling and identification of endocrine-metabolic systems. Theoretical aspects and their importance in practice. Math. Biosci., 72 (1984), 263-289.

[9] J. Cohen. Mathematics Is Biology's Next Microscope, Only Better; Biology Is Mathematics' Next Physics, Only Better. PLoS Biol., 2 (2004), e439. Published online 2004 December 14. doi: 10.1371/journal.pbio.0020439.

[10] J. Crow and M. Kimura. An Introduction to Population Genetics Theory. Harper \& Row, New York, 1970.

[11] M. Evans, N. Hastings, B. Peacock. Erlang distribution. Ch. 12 in Statistical Distributions, 3rd ed., Wiley, New York, 2000, 71-73.

[12] A. Ford. Modeling the environment. Island Press, 2010.

[13] W. Granta, J. Matisb, and T. Millerb. A Stochastic Compartmental Model for Migration of Marine Shrimp. Ecological Modeling, 54 (1991), 1-15.

[14] L. Gross. Quantitative training for life science students. BioScience, 44 (1994), 59. 
[15] P. Higgs. Frequency distributions in population genetics parallel those in statistical physics. Physical Review E, (1995), No. 51, 95-101.

[16] W. Hwang, Y. Cho, A. Zhang, M. Ramanathan. A novel functional module detection algorithm for protein-protein interaction networks. Algorithms for Molecular Biology (2006), No. 2, 24, doi:10.1186/1748-7188-1-24.

[17] J. Jacquez. Compartmental Analysis in Biology and Medicine, 3rd ed., Biomedware, Ann Arbor, MI, 1996.

[18] I. Karsai, G. Kampis. The crossroad between biology and mathematics: Scientific method as the basics of scientific literacy. BioScience, 60 (2010), 632-638.

[19] J. Knisley. Netlogo Migration Simulations, http://math.etsu.edu/symbiosis/migrations, (2011).

[20] J. Knisley, I. Karsai, A. Godbole, M. Helfgott, K. Joplin, E. Seier, D. Moore, H. Miller. Storytelling in the Symbiosis Project. To appear in Undergraduate Mathematics for the Life Sciences: Processes, Models, Assessment, and Directions, MAA Lecture Notes, 2010.

[21] J. Knisley. A 4-Stage Model of Mathematical Learning. The Mathematics Educator, 12 (2002), No. 1, 11-16.

[22] D. Lauffenburger. Receptors. Oxford University Press, Oxford, 1993.

[23] M. Malice, C. Lefevre. On Linear Stochastic Compartmental Models in Discrete Time. Bulletin of Mathematical Biology, 47 (1985), No. 2, 287-293.

[24] D. Moore, M. Helfgott, A. Godbole, K. Joplin, I. Karsai, J. Knisley, H. Miller, E. Seier. Creating Quantitative Biologists: The Immediate Future of SYMBIOSIS. To appear in Undergraduate Mathematics for the Life Sciences: Processes, Models, Assessment, and Directions, MAA Lecture Notes, 2010.

[25] J. Murray. Mathematical Biology. Springer, New York, 1989.

[26] W. Reed, B. Hughes. Theoretical size distribution of fossil taxa: analysis of a null model. Theoretical Biology and Medical Modeling, 4 (2007), 12, doi:10.1186/1742-4682-4-12

[27] L. Steen, ed. Math \& Bio 2010: Linking Undergraduate Disciplines. Mathematical Association of America, Washington, DC, 2005.

[28] D. Usher, T. Driscoll, P. Dhurjati, J. Pelesko, L. Rossi, G. Schleiniger, K. Pusecker, H. White. A transformative model for undergraduate quantitative biology education. CBE Life Sci Educ., 9 (2010), No. 3, 181-188.

[29] D. Welch, E. Rechisky, M. Melnychuk, A. Porter, C. Walters. Survival of Migrating Salmon Smolts in Large Rivers With and Without Dams. PLoS Biol, 6 (2008), No. 10, e265. doi:10.1371/journal.pbio.0060265. 
[30] U. Wilensky. NetLogo. http://ccl.northwestern.edu/netlogo/. Center for Connected Learning and Computer-Based Modeling, Northwestern University. Evanston, IL, (1999). 